\title{
Short and Medium-term Outcomes of Omphalocele and Gastroschisis: A Survey from a Tertiary Center
}

\section{Resultados a curto e médio prazo do onfalocelo e gastrosquisis: uma investigação de um centro terciário}

\author{
Alexandra Tavares Marques ${ }^{1 *(1)}$ José Estevão-Costa ${ }^{1,2 *(1)}$ \\ ${ }^{1}$ Department of Surgery and Physiology, Faculty of Medicine, \\ University of Porto, Porto, Portugal \\ 2 Pediatric Surgery Department, Centro Hospitalar Universitário de \\ São João, Porto, Portugal \\ ${ }^{3}$ Department of Pediatrics, Division of Neonatology, Faculty of \\ Medicine of Porto, Centro Hospitalar Universitário de São João, \\ Porto, Portugal
}

Henrique Soares ${ }^{30}$

Ana Catarina Fragoso ${ }^{1,2}$

\author{
Address for correspondence José Estevão-Costa, MD, PhD, \\ Department of Surgery and Physiology, Faculty of Medicine, \\ University of Porto, Alameda Prof. Hernâni Monteiro, 4200-319, \\ Porto, Portugal (e-mail: estevao@med.up.pt).
}

Rev Bras Ginecol Obstet 2022;44(1):10-18.

\begin{abstract}
Objective To characterize and compare the outcomes of omphalocele and gastroschisis from birth to 2 years of follow-up in a recent cohort at a tertiary center. Methods This is a retrospective clinical record review of all patients with gastroschisis and omphalocele admitted to the Neonatal Intensive Care Unit between January 2009 and December 2019.

Results There were 38 patients, 13 of whom had omphalocele, and 25 of whom had gastroschisis. Associated anomalies were present in 6 patients (46.2\%) with omphalocele and in $10(41.7 \%)$ patients with gastroschisis. Compared with patients with omphalocele, those with gastroschisis had younger mothers (24.7 versus 29.6 years; $p=0.033$ ), were born earlier ( 36 versus 37 weeks, $p=0.006$ ), had lower birth weight

Keywords

- omphalocele

- gastroschisis

- anomalies

- abdominal-wall defect

- morbidity $(2365 \pm 430.4$ versus $2944.2 \pm 571.9 \mathrm{~g} ; p=0.001)$, and had a longer hospital stay (24 versus 9 days, $p=0.001$ ). The neonatal survival rate was $92.3 \%$ for omphalocele and 91.7\% for gastroschisis. Thirty-four patients were followed-up over a median of 24 months; 13 patients with gastroschisis $(59.1 \%)$ and 8 patients with omphalocele (66.7\%) had at least one adverse event, mainly umbilical hernia (27.3\% vs $41.7 \%)$, intestinal obstruction ( $31.8 \%$ vs $8.3 \%$ ), or additional surgical interventions ( $27.3 \%$ vs $33.3 \%)$.
\end{abstract}

${ }^{*}$ Both authors have contributed equally to this work and share first authorship.

received

February 19, 2021

accepted

August 9, 2021
DOI https://doi.org/

10.1055/s-0041-1736299. ISSN 0100-7203.
(C) 2022. Federação Brasileira de Ginecologia e Obstetrícia. All rights reserved.

This is an open access article published by Thieme under the terms of the Creative Commons Attribution License, permitting unrestricted use, distribution, and reproduction so long as the original work is properly cited. (https://creativecommons.org/licenses/by/4.0/)

Thieme Revinter Publicações Ltda., Rua do Matoso 170, Rio de Janeiro, RJ, CEP 20270-135, Brazil 


\section{Resumo}

\author{
Palavras-chave \\ - onfalocele \\ - gastrosquisis \\ - anomalias \\ - defeito da parede \\ abdominal \\ - morbidade
}

Conclusion Despite the high proportion of prematurity, low birth weight, and protracted recovery, gastroschisis and omphalocele (without chromosomal abnormalities) may achieve very high survival rates; on the other hand, complications may develop in the first years of life. Thus, a very positive perspective in terms of survival should be transmitted to future parents, but they should also be informed that substantial morbidity may occur in the medium term.

Objetivo Caracterizar e comparar os desfechos do onfalocelo e gastrosquisis desde o nascimento até aos 2 anos de seguimento numa coorte recente de um centro terciário. Métodos Este é um estudo retrospectivo em que foi feita uma revisão dos registos clínicos de todos os pacientes com gastrosquisis e onfalocelo que foram internados na unidade de cuidados intensivos neonatais, entre janeiro de 2009 e dezembro de 2019. Resultados Identificamos 38 pacientes, 13 dos quais tinham onfalocelo e 25 dos quais tinham gastrosquisis. Anomalias associadas estavam presentes em 6 pacientes (46.2\%) com onfalocelo e 10 (41.7\%) com gastrosquisis. Comparativamente com os pacientes com onfalocelo, os pacientes com gastrosquisis tinham mães mais jovens (24.7 versus 29.6 anos; $p=0.033$ ), nasceram mais precocemente (36 versus 37 semanas, $p=0.006)$, com menor peso ao nascimento $(2,365 \pm 430.4$ versus $2,944.2 \pm 571.9 \mathrm{~g} ; p=0.001$ ), e o internamento teve uma duração mais longa (24 versus 9 dias, $p=0.001$ ). A taxa de sobrevivência neonatal foi de $92.3 \%$ para o onfalocelo e $91.7 \%$ para a gastrosquisis. Trinta e quatro pacientes foram seguidos durante um tempo mediano de seguimento de 24 meses: 13 com gastrosquisis (59.1\%) e 8 com onfalocelo (66.7\%) apresentaram pelo menos um evento adverso, sobretudo hérnia umbilical ( $27.3 \%$ vs $41.7 \%$ ), obstrução intestinal ( $31.8 \%$ vs $8.3 \%$ ) ou intervenções cirúrgicas adicionais (27.3\% vs $33.3 \%$ ).

Conclusão Apesar da alta proporção de prematuridade, de baixo peso e de recuperação lenta, os gastrosquisis, assim como os onfalocelos (sem anomalias cromossómicas), podem ter uma taxa de sobrevivência muito alta; por outro lado, nos primeiros anos de vida, podem surgir complicações não desprezíveis. Assim, aos futuros pais pode ser transmitida uma perspectiva muito positiva em termos de sobrevivência, embora eles também devam ser informados de que pode ocorrer morbidade substancial no médio prazo.

\section{Introduction}

Gastroschisis and omphalocele are the most common congenital abdominal wall defects, with a prevalence of $\sim 2$ to 4 per 10,000 live births, respectively. ${ }^{1}$ The etiology of these defects remains poorly understood. In contrast to omphalocele, the prevalence of gastroschisis has been increasing worldwide in recent decades. ${ }^{1}$

Omphalocele is a midline abdominal wall defect in which varying amounts of bowel and liver, and occasionally other organs, protrude through the base of the umbilicus into a membranous sac. The intestines are generally morphologically and functionally normal. ${ }^{2}$ Prognosis is determined mainly by the associated anomalies but also by the size of the defect and degree of liver exteriorization.,4 Omphalocele with co-occurring anomalies, which represents over $60 \%$ of cases of omphalocele, is associated with a high rate of pregnancy terminations and intrauterine fetal deaths and a significant rate of mortality in the neonatal period and at
1 year of age. ${ }^{3,5,6}$ Infants with isolated omphalocele have the best survival rate., ${ }^{5,6}$

Gastroschisis is as an abdominal wall defected, in which bowel and sometimes other abdominal organs herniate into the amniotic cavity without a membranous protective sac. The prognosis of infants with gastroschisis is primarily determined by the degree of intestinal injury. ${ }^{2,7}$ In contrast to omphalocele, the bowel in patients with gastroschisis is usually damaged due to chronic exposure to amniotic fluid, which results in a varying period of bowel dysmotility after birth, requiring prolonged parenteral nutrition and hospitalization. ${ }^{8-10}$ Associated anomalies are present in 8 to $34 \%$ of cases, most of them in the gastrointestinal tract. ${ }^{8,11}$ Extraintestinal and chromosomal abnormalities or recognizable syndromes are rare. ${ }^{8}$

Despite advances in prenatal diagnosis, surgical management and neonatal critical care over the last decades have resulted in improvement of survival rates of infants born with gastroschisis and omphalocele, the survivors are still at 
significant risk of short and long-term adverse outcomes. ${ }^{3,12}$ These include growth delay, neurodevelopmental deficiencies, recurrent abdominal pain, feeding difficulties, pulmonary insufficiency, recurrent infections, umbilical revision for hernia or scar repair, bowel obstruction requiring surgical treatment, and other surgical problems. ${ }^{3,13}$ Efforts have been made to identify prenatal risk factors associated with gastroschisis and omphalocele, ${ }^{14}$ the optimal timing and route of delivery, ${ }^{15,16}$ immediate $v s$ staged repair, ${ }^{17,18}$ and other factors that may be amenable to improve outcomes as well to reduce the treatment costs. However, many of these issues still lacks consensus. ${ }^{7,8,10}$

The aim of the present study was to retrospectively review recent live births with gastroschisis and omphalocele, managed at our tertiary care center, regarding demographic features, associated anomalies, neonatal outcomes, and progress of the survivors until 2 years of age.

\section{Methods}

This is a retrospective study of all patients with gastroschisis and omphalocele that were born alive and were admitted to the Neonatal Intensive Care Unit of Centro Hospitalar Universitário de São João (CHUSJ), a tertiary center in Portugal, between January 2009 and December 2019. Elective terminations, spontaneous miscarriages, and intrauterine fetal deaths were excluded from our study. The present study received approval from the ethics committee of CHUSJ.

All data were obtained from the medical records of patients. Maternal information included age, parity, and previous abortions. Obstetric data included diagnosis (if prenatal, gestational age) and complications (amniotic fluid volume alterations, intrauterine growth restriction, gestational diabetes, and other). For the purposes of this study, intrauterine growth restriction (IUGR) was defined as birth weight below the $10^{\text {th }}$ percentile. Gestational age at birth, mode of delivery, gender, birth weight, resuscitation, meconium aspiration, APGAR scores, herniated organs, time and type of surgical repair, and other surgeries during the first hospitalization were also obtained.

The morbidity indicators evaluated were length of hospital stay, duration of parenteral nutrition, time to initiate enteral feeding, need of mechanical ventilation, ventilator duration, complications (intestinal occlusion, necrotizing enterocolitis, and sepsis among others), and discharge with parenteral nutrition. Data of co-occurring major anomalies were also collected. We defined a major congenital anomaly as an anomaly that create significant medical problems for the patient or that require specific surgical or medical management. ${ }^{19,20}$ Anomalies considered physiologic (e.g., patent foramen ovale), transient or minor, were excluded. The classification of omphaloceles in giant/large or small was based on the defect size and on the qualitative descriptions ("small," "large" and "giant") used by pediatric surgeons in surgical reports. Giant omphalocele was defined as a defect with a diameter greater than $5 \mathrm{~cm}$ and/or containing greater than $75 \%$ of the liver in the sac. ${ }^{3}$ Complex gastroschisis was defined as gastroschisis associated with at least one of the following intestinal complications: atresia, volvulus, perforation, and necrosis.

The follow-up during the first 2 years of life focused on the following variables: presence of umbilical hernia, intestinal obstruction, surgical interventions, need for parenteral nutrition, need for supplemental oxygen, and anthropometric parameters (weight and length).

The outcomes of patients born with gastroschisis were compared with those of patients born with omphalocele. Statistical analysis was performed using the IBM SPSS Statistics for Windows, Version 27.0 (IBM Corp., Armonk, NY, USA). Continuous variables were expressed as mean ( \pm standard deviation) or median (P25-P75), in normal or skewed distribution, respectively. Categorical variables were described by absolute (n) and relative frequencies (\%). For comparisons, parametric (independent $t$-test) and nonparametric (Mann-Whitney U test), tests were used for continuous variables with normal or skewed distribution, respectively. The Fisher exact test was used for comparisons involving categorical variables. Statistical significance was set at $5 \%$. This study is reported in line with the STROBE checklist for observational studies. ${ }^{21}$

\section{Results}

There was a total of 38 patients, 13 with omphalocele and 25 with gastroschisis. All but two (one with omphalocele and one with gastroschisis) were diagnosed prenatally. One newborn with gastroschisis was transferred to another hospital on the $6^{\text {th }}$ day of life and not subsequently followed-up at our institution.

Maternal and neonatal demographic data are summarized in - Table 1. Maternal age was significantly lower for gastroschisis. Although not reaching statistical significance, there was a higher proportion of primigravida mothers in the gastroschisis group. There was no significant difference between omphalocele and gastroschisis regarding mother's history of previous abortions either.

There were 2 (5.3\%) multiple pregnancies, both with twins, discordant for the defect (1 with gastroschisis and 1 with omphalocele). Abnormal amniotic fluid volume (oligohydramnios or polyhydramnios) and IUGR were present in 2 (15.4\%) and 3 (23.1\%) omphalocele pregnancies, respectively. For the 22 gastroschisis pregnancies with available fetal ultrasound information, 2 (9.1\%) had abnormal amniotic fluid volume and 7 (31.8\%) had IUGR.

Two patients with omphalocele were delivered vaginally, while the other patients were delivered by elective or emergency cesarean section. At birth, patients with gastroschisis presented a significantly lower gestational age than patients with omphalocele. This reflects the higher proportion of preterm deliveries associated with gastroschisis (80\%) when compared with omphalocele (53.8\%). Birth weight was significantly lower for patients with gastroschisis than for those with omphalocele. For both defects, there was a similar preponderance of males.

Visceral content exteriorized through the abdominal wall defect at birth and associated anomalies are shown 
Table 1 Maternal and neonatal demographics

\begin{tabular}{|c|c|c|c|c|}
\hline & $\begin{array}{l}\text { Total } \\
(n=38)\end{array}$ & Omphalocele $(n=13)$ & Gastroschisis $(n=25)$ & $P$-value \\
\hline Maternal age (years) & $26.4 \pm 6.8$ & $29.6 \pm 5.8$ & $24.7 \pm 6.8$ & 0.033 \\
\hline Gestational age at diagnosis (weeks) & $20[13-24]$ & $21[12-22]$ & 19 [13-27] & 0.803 \\
\hline Primigravida & $25(65.8 \%)$ & $7(53.8 \%)$ & $18(72 \%)$ & 0.301 \\
\hline Previous abortions & $10(26.3 \%)$ & $4(30.8 \%)$ & $6(25 \%)$ & 0.709 \\
\hline Gestational age at delivery (weeks) & $36[35-37]$ & $37[36-38]$ & $36[34-36]$ & 0.006 \\
\hline \multicolumn{5}{|l|}{ Mode of delivery } \\
\hline vaginal & $2(5.3 \%)$ & $2(15.4 \%)$ & 0 & 0.111 \\
\hline cesarean section & $36(94.7 \%)$ & $11(84.6 \%)$ & $25(100 \%)$ & \\
\hline \multicolumn{5}{|l|}{ Gender } \\
\hline Male & $23(60.5 \%)$ & $7(53.8 \%)$ & $16(64 \%)$ & 0.728 \\
\hline Female & $15(39.5 \%)$ & $6(46.2 \%)$ & $9(36 \%)$ & \\
\hline Birth weight (grams) & $2,563.4 \pm 551.1$ & $2,944.2 \pm 571.9$ & $2,365 \pm 430.4$ & 0.001 \\
\hline
\end{tabular}

Values are given as mean $\pm \mathrm{SD}$, median [P25 - P75] or $\mathrm{n}(\%)$.

Table 2 Viscera exteriorized and associated anomalies

\begin{tabular}{|c|c|c|c|}
\hline & $\begin{array}{l}\text { Total } \\
(n=38)\end{array}$ & Omphalocele $(n=13)$ & Gastroschisis $(n=25)$ \\
\hline \multicolumn{4}{|l|}{ Viscera exteriorized } \\
\hline Bowel (only) & 20 (52.6\%) & $6(46.1 \%)$ & $14(56 \%)$ \\
\hline Liver (only) & $3(7.9 \%)$ & $3(23.1 \%)$ & - \\
\hline Bowel + liver & $3(7.9 \%)$ & $3(23.1 \%)$ & - \\
\hline Bowel + liver + stomach + spleen & $1(2.6 \%)$ & $1(7.7 \%)$ & - \\
\hline Bowel + stomach & $5(13.2 \%)$ & - & $5(20 \%)$ \\
\hline Bowel + bladder and/or gonads & $6(15.8 \%)$ & - & $6(24 \%)$ \\
\hline Associated anomalies, total * & $16(43.2 \%)$ & $6(46.2 \%)$ & $10(41.7 \%)$ \\
\hline Beckwith-Wiedemann syndrome & $2(5.4 \%)$ & $2(15.4 \%)$ & - \\
\hline Gastrointestinal anomalies, total & $6(16.2 \%)$ & - & $6(25 \%)$ \\
\hline Bowel atresia & $3(8.1 \%)$ & - & $3(12.5 \%)$ \\
\hline Enteric duplication cyst & $1(2.7 \%)$ & - & $1(4.2 \%)$ \\
\hline Meckel diverticulum & $2(5.4 \%)$ & - & $2(8.3 \%)$ \\
\hline Cardiac anomalies, total & $3(8.1 \%)$ & $2(15.4 \%)$ & $1(4.2 \%)$ \\
\hline VSD & $1(2.7 \%)$ & $1(7.7 \%)$ & - \\
\hline ASD & $2(5.4 \%)$ & $1(7.7 \%)$ & $1(4.2 \%)$ \\
\hline Int-IVC & $1(2.7 \%)$ & $1(7.7 \%)$ & - \\
\hline Central nervous system anomalies, total & $4(10.8 \%)$ & $3(23.1 \%)$ & $1(4.2 \%)$ \\
\hline ACC & $1(2.7 \%)$ & $1(7.7 \%)$ & - \\
\hline Nasal glial heterotopy & $1(2.7 \%)$ & $1(7.7 \%)$ & - \\
\hline Hydrocephaly & $1(2.7 \%)$ & - & $1(4.2 \%)$ \\
\hline Microcephaly & $1(2.7 \%)$ & $1(7.7 \%)$ & - \\
\hline Cryptorchidism & $3(8.1 \%)$ & $1(7.7 \%)$ & $2(8.3 \%)$ \\
\hline Hydronephrosis & $1(2.7 \%)$ & - & $1(4.2 \%)$ \\
\hline Syndactyly & $2(5.4 \%)$ & - & $2(8.3 \%)$ \\
\hline
\end{tabular}

Abbreviations: ACC, agenesis of the corpus callosum; ASD, atrial septal defect; Int-IVC, interrupted inferior vena cava; VSD, ventral septal defect Values are given as $\mathrm{n}(\%)$

${ }^{*} n=24$ for associated anomalies with gastroschisis due to missing information from 1 patient transferred to another hospital 
14 Short and Medium-term Outcomes of Omphalocele and Gastroschisis Marques et al.

Table 3 Comparison of clinical outcomes between patients with omphalocele and gastroschisis

\begin{tabular}{|c|c|c|c|c|}
\hline & $\begin{array}{l}\text { Total } \\
(n=38)\end{array}$ & Omphalocele $(n=13)$ & Gastroschisis $(n=25)$ & $P$-value \\
\hline Resuscitation at birth & $6(15.8 \%)$ & $3(23.1 \%)$ & $3(12.0 \%)$ & 0.392 \\
\hline \multicolumn{5}{|l|}{ APGAR Score } \\
\hline $1^{\text {st }}$ minute $<7$ & $4(10.5 \%)$ & $2(15.4 \%)$ & $2(8 \%)$ & 0.595 \\
\hline $5^{\text {th }}$ minute $<7$ & $2(5.3 \%)$ & $1(7.7 \%)$ & $1(4 \%)$ & 1.000 \\
\hline \multicolumn{5}{|l|}{ Type of surgery } \\
\hline Primary closure & $33(86.8 \%)$ & $12(92.3 \%)$ & $21(84 \%)$ & 0.643 \\
\hline Staged closure (silo) & $5(13.2 \%)$ & $1(7.7 \%)$ & $4(16 \%)$ & \\
\hline More than 1 surgery during hospital stay ${ }^{*}, \pi$ & $7(18.9 \%)$ & $2(15.4 \%)$ & $5(20.8 \%)$ & 1.000 \\
\hline Hospital stay (days) * & $20[11.5-31.5]$ & $9[5.5-17.5]$ & $24[15.8-36.8]$ & 0.001 \\
\hline $\begin{array}{l}\text { Need for mechanical ventilation } \\
\text { Ventilator duration (days) }\end{array}$ & $\begin{array}{l}34(89.5 \%) \\
3[1.8-5]\end{array}$ & $\begin{array}{l}11(84.6 \%) \\
2[1-3.5]\end{array}$ & $\begin{array}{l}23(92 \%) \\
4[2-6]\end{array}$ & $\begin{array}{l}0.595 \\
0.052\end{array}$ \\
\hline Parenteral nutrition duration (days) * & $16[9-27.5]$ & $8[3-13.5]$ & $22.5[14-34.5]$ & $<0.001$ \\
\hline Initiation of enteral feeding (days of life) * & $9.5[4.8-14.5]$ & $4[2-5.5]$ & $12[9.5-19.5]$ & $<0.001$ \\
\hline \multicolumn{5}{|l|}{ Complications during hospital stay, total* } \\
\hline Sepsis & $8(21.6 \%)$ & $1(7.7 \%)$ & $7(29.2 \%)$ & 0.216 \\
\hline Intestinal occlusion & $2(5.4 \%)$ & 0 & $2(8.3 \%)$ & 0.532 \\
\hline Neonatal death * & $3(8.1 \%)$ & $1(7.7 \%)$ & $2(8.3 \%)$ & 1.000 \\
\hline
\end{tabular}

Values are given as median [P25-P75] or $\mathrm{n}(\%)$

${ }^{*} n=24$ for gastroschisis due to missing information from 1 patient transferred to another hospital

"For omphalocele: silo placement after failed primary closure $(n=1)$, inguinal hernia repair and orchidopexy $(n=1)$. For gastroschisis: silo removal with or without intestinal atresia repair $(n=3)$, surgery for adhesion-related bowel obstruction $(n=1)$, surgical wound debridement $(n=1)$, intestinal atresia repair $(n=1)$

in -Table 2. Associated anomalies were present in 6 patients (46.2\%) with omphalocele and in 10 (41.7\%) with gastroschisis. Two male patients with gastroschisis had balanced translocations ( $\mathrm{t}[6 ; 8][\mathrm{q} 13 ; \mathrm{p} 23.1]$ and $\mathrm{t}[2 / 9]$ ) that were not associated with any known phenotype, and, therefore, were not included in the analysis. The translocation $t(6 ; 8)(q 13$; p23.1) was likely associated with infertility since the mother had had 5 previous pregnancy losses. Anomalies in the gastrointestinal tract were found only in patients with gastroschisis, the majority being bowel atresias. Of the 6 patients (24\%) with complex gastroschisis, 2 presented with bowel atresia only, 1 presented with bowel atresia and perforation, and the other 3 presented with bowel perforation either alone or with other complications that define complex gastroschisis.

All patients with omphalocele had a normal karyotype. Genetic syndromes were found exclusively in patients with omphalocele (2 patients diagnosed with Beckwith-Wiedemann syndrome). There was a third patient with omphalocele and multiple anomalies in several organs, including one hemivertebrae, several cardiac malformations (mesocardia, ASD, interrupted inferior vena cava with azygos continuation to superior vena cava), and several facial abnormalities associated with Duane type 1 syndrome. There were 4 omphaloceles considered giant (30.8\%), 3 of which had no associated anomalies. No patient had rupture of the omphalocele sac. One patient with a small omphalocele presented with ileum stenosis at birth.
Most patients had surgical repair of the abdominal wall defect on the first day of life, regardless of the type of the defect. Only one patient with omphalocele had surgery on the second day of life. Primary closure was achieved in $86.8 \%$ of the patients (-Table 3 ). Two patients ( 1 with complex gastroschisis and 1 with giant omphalocele) required silo placement after failed primary repair due to abdominal compartment syndrome. Both have died. For the other 3 patients ( 1 patient with complex and 2 with simple gastroschisis, respectively) who underwent staged closure, the mean time with silo was 10.7 days. Two patients with large omphaloceles required the use of a polytetrafluoroethylene (PTFE) patch. Intestinal atresia was repaired either in a second operation after primary closure (1 patient with complex gastroschisis) or during secondary abdominal closure (1 patient with complex gastroschisis). Intestinal stenosis (1 patient with complex gastroschisis and 1 patient with omphalocele) was repaired during primary closure.

Overall, patients with gastroschisis had a higher rate of complications during the hospital stay, as shown in - Table 3 . Sepsis and intestinal occlusion were more frequent, but without statistical significance, in patients with gastroschisis. Necrotizing enterocolitis was not found in any of the patients. Gastroschisis was significantly associated with a longer duration of parenteral nutrition and longer time to initiate enteral feeding. Patients with gastroschisis were hospitalized twice as long as patients with omphalocele. One of the patients with complex gastroschisis (bowel 
Table 4 Adverse outcomes during the follow-up

\begin{tabular}{lllll}
\hline & $\begin{array}{l}\text { Total } \\
(\boldsymbol{n}=34)\end{array}$ & Omphalocele $(\boldsymbol{n}=12)$ & Gastroschisis $(\boldsymbol{n}=\mathbf{2 2 )}$ & $P$-value \\
\hline Patients with adverse outcomes & $21(61.8 \%)$ & $8(66.7 \%)$ & $13(59.1 \%)$ & 0.727 \\
Umbilical hernia & $11(32.4 \%)$ & $5(41.7 \%)$ & $6(27.3 \%)$ & 0.459 \\
Intestinal occlusion episodes & $8(23.5 \%)$ & $1(8.3 \%)$ & $7(31.8 \%)$ & 0.210 \\
Surgical interventions ${ }^{\pi}$ & $10(29.4 \%)$ & $4(33.3 \%)$ & $6(27.3 \%)$ & 0.714 \\
Parenteral nutrition & $1(2.9 \%)$ & 0 & $1(4.5 \%)$ & 1.000 \\
Supplemental oxygen & $1(2.9 \%)$ & $1(8.3 \%)$ & 0 & 0.353 \\
\hline
\end{tabular}

Values are given as $\mathrm{n}(\%)$

"The surgical interventions are listed in - Supplementary Table S1

perforation only), who had an associated enteric duplication cyst, underwent a massive bowel resection during the primary repair surgery and developed ultra-short gut syndrome. One patient with Beckwith-Wiedemann syndrome, who had been born preterm (33 weeks) and with bronchopulmonary dysplasia, was discharged on nasogastric feeds and supplemental oxygen.

The neonatal survival rate was $92.3 \%$ for omphalocele and 91.7\% for gastroschisis. All the three deaths occurred in the neonatal period and involved preterm neonates. Two deaths were of patients with gastroschisis. One of them presented complex gastroschisis with bowel and bladder exteriorized; the patient underwent silo placement and died on day 3 of life. The second patient was born by cesarean section in another hospital and suffered perinatal asphyxia. The death occurred on day 16 of life and was related with severe hypoxic-ischemic encephalopathy. The third patient was a twin born with a giant omphalocele containing the bowel, stomach, liver, and spleen. After failed primary closure, a silo was applied, but the patient died on day 3 of life.

Thirty-four patients were followed-up, with a median follow-up time of 24 months (range 11-24), and the outcomes are summarized in -Table 4 . Thirteen patients with gastroschisis (59.1\%) and 8 patients with omphalocele (66.7\%) had at least one adverse event during the followup. Ten patients (29.4\%) needed further surgical interventions due to diverse comorbidities, and the details are given in - Supplementary Table $\mathbf{S 1}$ of the supplementary material. Seven patients with gastroschisis (31.8\%) suffered intestinal obstruction episodes, with adhesions being the cause in 3 of them. Of the 5 surviving patients with complex gastroschisis, 3 underwent surgery for bowel obstruction during the follow-up period. Only 1 patient with omphalocele (8.3\%) had bowel obstruction, and it was caused by an intestinal volvulus. The patient with complex gastroschisis that had ultra-short gut syndrome remained hospitalized for 788 days due to several complications (intestinal obstruction episodes and catheter-related infections) and lack of family support. Although it was possible to make progress to oral feeding, it was not possible to wean parenteral nutrition; therefore, one serial transverse enteroplasty (STEP) procedure was performed at 10 months. Despite that, the patient continued to be dependent on parenteral nutrition with irregular enteral tolerance during the follow-up period of our study.
The patient with Beckwith-Wiedemann syndrome and bronchopulmonary dysplasia received supplemental oxygen until 1 year of age.

Anthropometric data at 2 years of follow-up was available for 7 patients with omphalocele and 15 patients with gastroschisis. Only 1 patient with omphalocele (patient 2 in -Supplementary Table S1) that had been born with both weight and length below the $10^{\text {th }}$ percentile was still below that percentile at 2 years of age. Of the 15 patients with gastroschisis, 3 had both weight and length at birth below the $10^{\text {th }}$ percentile, 1 had birth length below the $10^{\text {th }}$ percentile, and 1 had birth weight below the $10^{\text {th }}$ percentile. Only 1 patient with gastroschisis had the length below the $10^{\text {th }}$ percentile at 2 years of age, indicating improvement in the growth between birth and follow-up for most children.

\section{Discussion}

This study examines the detailed short and medium-term outcome of 38 live births with omphalocele and gastroschisis. This represents approximately an institutional prevalence of 10 cases per 10,000 live births; however, our center is a referral one for antenatal diagnosis and therapy; that figure is probably indicative of an estimated prevalence of 3 to 5 cases per 10,000 live births in the country, as reported by others. 5,22 The etiology of these anterior abdominal wall defects and the associated risk factors remain controversial. Young maternal age has been recognized as one of the strongest risk factors for gastroschisis. ${ }^{1,14}$ Consistent with this, the mothers of fetuses with gastroschisis were markedly younger (average 24.7 years) than mothers with fetuses with omphalocele (29.6 years) in our cohort of patients. For omphalocele, both young and advanced maternal age have been identified as risk factors. ${ }^{5,14}$

The majority of patients in our cohort of cases were delivered at preterm. Gastroschisis patients were born earlier and with lower birth weight than those with omphalocele, which is consistent with other studies. ${ }^{11,23}$ All patients with gastroschisis and $84.6 \%$ of the patients with omphalocele were delivered by caesarean section. The observed rate for cesarean delivery was high, and this is mainly explained by the fact that a planned delivery in a tertiary care center, with access to appropriate neonatal and pediatric surgical services, is more readily achieved by caesarean section than by 
vaginal delivery. There is also a fear of some obstetricians of inducing birth dystocia or injury of the exposed abdominal viscera during vaginal delivery. ${ }^{7}$ However, several studies failed to demonstrate that cesarean section is superior to vaginal delivery for neonates with omphalocele and gastroschisis. $^{7,22,24}$

The choice of the method to reduce the exteriorized organs and close the fascial defect depends on several factors, namely the size of the defect, patient stability, gestational maturity, and the presence of associated anomalies. Many surgeons recommend primary closure, when feasible, to be done within a few hours after birth. ${ }^{8}$ This is also the preferred strategy at our center. Our primary abdominal closure rate of $86.8 \%$ is similar to other published rates. ${ }^{4,23,25}$ Staged repair with silo placement may be preferable for the unstable neonate, large defects and when gastroschisis is associated with significant intestinal damage. ${ }^{4,7}$ In our study, one patient with a giant omphalocele and one patient with a large and complex gastroschisis developed compartment syndrome after primary closure and were converted to a staged reduction. Both patients died. This highlights the difficulties in managing patients with large defects as there is lack of strong evidence for decision-making. According to Lee et al., ${ }^{26}$ initial primary closure should never be attempted for giant omphaloceles, while other studies report that immediate closure with or without the use of a synthetic patch is possible. ${ }^{4,27}$

The length of hospitalization was significantly longer for patients with gastroschisis than for patients with omphalocele. The factors that contributed to this difference included the later onset of bowel function in patients with gastroschisis, as indicated by their significantly longer time in parenteral nutrition and to initiate enteral feeding, compared with patients with omphalocele. Furthermore, although not reaching statistical significance, patients with gastroschisis were more likely to have complications during hospitalization, namely sepsis. Our findings are in line with other studies that have shown that patients with gastroschisis generally have a more complicated neonatal course than those with omphalocele. ${ }^{11}$ This is related to the expected bowel dysmotility and deficient nutrient absorption associated with gastroschisis, while patients with omphalocele typically have a normal or less impaired bowel function after birth. ${ }^{2}$ Therefore, a longer period of parenteral nutrition is generally required for infants with gastroschisis while they wait for the recovery of gut function. During this period, patients are at risk of having complications associated with the parenteral nutrition, namely catheter-related sepsis, and liver disease. ${ }^{28}$ In our cohort of patients with gastroschisis, $24 \%$ had complex gastroschisis, which is in line with other studies. ${ }^{29}$ These patients typically have worse outcomes, and our findings confirm this.

Patients with omphalocele are more frequently diagnosed with associated anomalies compared with those with gastroschisis. ${ }^{7,11}$ However, in our cohort of patients, we found that the proportion of patients diagnosed with associated anomalies was similar in omphalocele (46.2\%) and in gastroschisis (41.7\%). Given that elective terminations and intrauterine fetal deaths were excluded from our study, the proportion of patients with omphalocele and associated anomalies is lower than otherwise would have been expected. This also explains the absence of omphalocele cases with chromosomal defects. Similarly, Heider et al. ${ }^{30}$ found that by excluding elective terminations and spontaneous miscarriages, the rate of associated anomalies in a cohort of 36 cases of omphalocele was $31 \%$. We found a pattern of anomalies associated with each defect similar to what is reported in the literature. ${ }^{6,8,11,31}$ Patients with omphalocele were more likely to have genetic syndromes and central nervous system anomalies, while gastrointestinal anomalies were more commonly associated with gastroschisis.

Survival rates greater than $90 \%$ have been reported for gastroschisis. ${ }^{12,32}$ Our data corroborates this finding. We also report a high survival rate $(92.3 \%)$ for omphalocele, and this is explained by the absence of cases with abnormal karyotype and the high proportion of isolated omphaloceles (53.8\%) in our cohort of cases. Previously, Marshall et al. ${ }^{5}$ reported a survival rate of $92 \%$ for patients with isolated omphalocele, and this value decreased to $38.8 \%$ for patients with chromosomal anomalies. Furthermore, excluding one case with bronchopulmonary dysplasia, none of the other survivors with omphalocele had significant cardiopulmonary complications during hospital stay. Thus, our group of patients with omphalocele may represent a selected subset of cases with low postoperative morbidity and mortality.

Medium-term morbidity was evaluated through examination of the outcomes listed in - Table 4 for a median follow-up of 24 months, and through evaluation of the anthropometric parameters at 2 years of age. Infants with omphalocele and with gastroschisis typically show an early growth delay that improves over time in most cases. $3,10,20$ We also observed an improvement of growth parameters from birth to 2 years of age, such that only 2 patients ( 1 with omphalocele and 1 with gastroschisis) had length and/or weight below the $10^{\text {th }}$ percentile at 2 years of age.

None of the outcomes in -Table 4 were significantly different between gastroschisis and omphalocele. A higher proportion of patients with gastroschisis (31.8\% versus $8.3 \%$ ) had bowel obstruction during the follow-up period, mainly due to intestinal adhesions. Adhesive small bowel obstruction is a frequent and serious complication in the first year of life, particularly in patients with gastroschisis. ${ }^{12,33}$ Van Eijck et al. ${ }^{33}$ reported a series of 55 patients with gastroschisis and 92 patients with omphalocele, in which $25 \%$ and $13 \%$, respectively, developed adhesive small bowel obstruction. The higher propensity of gastroschisis for adhesive small bowel obstruction is postulated to be a consequence of the inflammatory reaction of the bowel serosa following prolonged exposure to the amniotic fluid, and the hypoperistalsis that characterizes the newborns with gastroschisis also favors adhesion formation. ${ }^{33}$

One of the most severe complications of anterior abdominal wall defects is short gut syndrome, which is the most common cause of intestinal failure in children. ${ }^{34}$ Bowel atresia and other gastrointestinal anomalies, midgut volvulus, or necrotizing enterocolitis can predispose patients with 
gastroschisis and omphalocele to short bowel syndrome., ${ }^{7,10}$ This condition can be handled with pharmacologic interventions and intestinal lengthening procedures with the ultimate goal of avoiding intestinal transplantation. In our study, one patient with complex gastroschisis who developed ultra-short gut syndrome and underwent one STEP procedure at 10 months was still dependent on total parenteral nutrition at 2 years of age.

Umbilical hernia is a well-known complication following repair of congenital abdominal defects. ${ }^{12,35}$ The presence of umbilical hernia, abdominal scars or the lack of an umbilicus is known to cause psychosocial stress in some children and their parents. ${ }^{13,23}$ Henrich et al. ${ }^{23}$ reported a series of 15 patients with omphalocele and 20 patients with gastroschisis in whom $20 \%$ and $15 \%$, respectively, developed a ventral hernia. In our study, we found a high proportion of patients with omphalocele (41.7\%) and gastroschisis (27.3\%) who developed an umbilical hernia. An expectant approach may be considered as the hernia resolves spontaneously in many patients. ${ }^{35}$ In our study, only one patient underwent hernia repair during the follow-up period. For gastroschisis, the most consistent risk factor for umbilical hernia is sutureless closure, ${ }^{35}$ but this method is not used at our center. Hawkins et al. ${ }^{17}$ compared primary closure with SILO closure and found an equivalent hernia rate between primary closure and longer duration of SILO reduction, with SILO placed for less than 5 days associated with the lowest incidence of ventral hernia. ${ }^{17}$ Given that, in our study, a small number of patients (3 survivors) underwent secondary closure, we cannot take conclusions about the impact of the repair method on the development of umbilical hernia.

Our results support a hopeful information in terms of survival to be transmitted to future parents of a child born with gastroschisis or with omphalocele with a normal karyotype. However, during prenatal counseling, parents should be informed that neonates with gastroschisis and omphalocele are at risk for serious complications, including longer duration of parenteral feeding, presence of other anomalies requiring more urgent treatment (e.g., bowel atresia), need of reoperation and prolonged hospital stays. Furthermore, some survivors may experience a non-negligible morbidity in the first 2 years of life, as explained above.

There are some limitations to our study, which include those of any retrospective analysis, such as selection bias, potential confounders, incomplete or missing data, and unmeasured factors that could have changed the results. In addition, this study does not contemplate all the mortality commonly associated with omphalocele because we included only live births. The sample is relatively small, but it is from a single center with standardized procedures of care, allowing a comprehensive and detailed characterization of the natural history and outcomes of these abdominal wall defects.

\section{Conclusion}

Our study presents a comprehensive overview of the management and outcome of live births with gastroschisis and omphalocele in a large tertiary center. The present study corroborates that, when compared with omphalocele, patients with gastroschisis had younger mothers, lower gestational age and weight at birth, and a more prolonged hospitalization course. Both gastroschisis and omphalocele (with no chromosomal abnormalities) were associated with survival rates greater than $90 \%$; however, significant morbidity may occur in the medium term.

\section{Contributors}

All the authors participated in the concept and design of the present study. Marques A. T. and Estevão-Costa J. have performed the analysis and interpretation of data and the writing of the article. All the authors contributed to critical review of the intellectual content and have approved the manuscript as submitted.

\section{Conflict of Interests}

The authors have no conflict of interests to declare.

\section{References}

1 Stallings EB, Isenburg JL, Short TD, et al. Population-based birth defects data in the United States, 2012-2016: A focus on abdominal wall defects. Birth Defects Res. 2019;111(18):1436-1447. Doi: $10.1002 /$ bdr2.1607

2 Langer JC. Abdominal wall defects. World J Surg. 2003;27(01): 117-124. Doi: 10.1007/s00268-002-6746-1

3 Baerg JE, Munoz AN. Long term complications and outcomes in omphalocele. Semin Pediatr Surg. 2019;28(02):118-121. Doi: 10.1053/j.sempedsurg.2019.04.004

4 Raymond SL, Downard CD, St Peter SD, et al. Outcomes in omphalocele correlate with size of defect. J Pediatr Surg. 2019; 54(08):1546-1550. Doi: 10.1016/j.jpedsurg.2018.10.047

5 Marshall J, Salemi JL, Tanner JP, et al; National Birth Defects Prevention Network. Prevalence, correlates, and outcomes of omphalocele in the United States, 1995-2005. Obstet Gynecol. 2015;126(02):284-293. Doi: 10.1097/aog.0000000000000920

6 Nembhard WN, Bergman JEH, Politis MD, et al. A multi-country study of prevalence and early childhood mortality among children with omphalocele. Birth Defects Res. 2020;112(20):1787-1801. Doi: 10.1002/bdr2.1822

7 Christison-Lagay ER, Kelleher CM, Langer JC. Neonatal abdominal wall defects. Semin Fetal Neonatal Med. 2011;16(03):164-172. Doi: $10.1016 /$ j.siny.2011.02.003

8 Ledbetter DJ. Gastroschisis and omphalocele. Surg Clin North Am. 2006;86(02):249-260, vii. Doi: 10.1016/j.suc.2005.12.003

9 Correia-Pinto J, Tavares ML, Baptista MJ, et al. Meconium dependence of bowel damage in gastroschisis. J Pediatr Surg. 2002;37 (01):31-35. Doi: 10.1053/jpsu.2002.29422

10 Bhat V, Moront M, Bhandari V. Gastroschisis: a state-of-the-art review. Children (Basel). 2020;7(12):302. Doi: 10.3390/children7120302

11 Corey KM, Hornik CP, Laughon MM, McHutchison K, Clark RH, Smith PB. Frequency of anomalies and hospital outcomes in infants with gastroschisis and omphalocele. Early Hum Dev. 2014;90(08):421-424. Doi: 10.1016/j.earlhumdev.2014.05.006

12 Harris EL, Minutillo C, Hart S, et al. The long term physical consequences of gastroschisis. J Pediatr Surg. 2014;49(10): 1466-1470. Doi: 10.1016/j.jpedsurg.2014.03.008

13 Suominen J, Rintala R. Medium and long-term outcomes of gastroschisis. Semin Pediatr Surg. 2018;27(05):327-329. Doi: 10.1053/j.sempedsurg.2018.08.008

14 Frolov P, Alali J, Klein MD. Clinical risk factors for gastroschisis and omphalocele in humans: a review of the literature. Pediatr Surg Int. 2010;26(12):1135-1148. Doi: 10.1007/s00383-010-2701-7 
15 Gelas T, Gorduza D, Devonec S, et al. Scheduled preterm delivery for gastroschisis improves postoperative outcome. Pediatr Surg Int. 2008;24(09):1023-1029. Doi: 10.1007/s00383-008-2204-y

16 Logghe HL, Mason GC, Thornton JG, Stringer MD. A randomized controlled trial of elective preterm delivery of fetuses with gastroschisis. J Pediatr Surg. 2005;40(11):1726-1731. Doi: 10.1016/j.jpedsurg.2005.07.047

17 Hawkins RB, Raymond SL, St Peter SD, et al. Immediate versus silo closure for gastroschisis: Results of a large multicenter study. J Pediatr Surg. 2020;55(07):1280-1285. Doi: 10.1016/j.jpedsurg.2019.08.002

18 Skarsgard ED. Immediate versus staged repair of omphaloceles. Semin Pediatr Surg. 2019;28(02):89-94. Doi: 10.1053/j.sempedsurg.2019.04.010

19 Uhlmann WR, Schuette JL, Yashar B. A guide to genetic counseling. 2nd ed. Hoboken: Wiley-Blackwell; 2009

20 Valente L, Pissarra S, Henriques-Coelho T, Flor-de-Lima F, Guimarães H. Gastroschisis: factors influencing 3-year survival and digestive outcome. J Pediatr Neonatal Individual Med. 2015;5 (01):e050114. Doi: 10.7363/050114(JPNIM)

21 von Elm E, Altman DG, Egger M, Pocock SJ, Gøtzsche PC, Vandenbroucke JPSTROBE Initiative. Strengthening the Reporting of Observational Studies in Epidemiology (STROBE) statement: guidelines for reporting observational studies. BMJ. 2007;335 (7624):806-808. Doi: 10.1136/bmj.39335.541782.AD

22 Calcagnotto H, Müller AL, Leite JC, et al. [Associated factors for perinatal mortality in gastroschisis]. Rev Bras Ginecol Obstet. 2013;35(12): 549-553. Doi: 10.1590/s0100-72032013001200004 Portuguese.

23 Henrich K, Huemmer HP, Reingruber B, Weber PG. Gastroschisis and omphalocele: treatments and long-term outcomes. Pediatr Surg Int. 2008;24(02):167-173. Doi: 10.1007/s00383-007-2055-y

24 Kirollos DW, Abdel-Latif ME. Mode of delivery and outcomes of infants with gastroschisis: a meta-analysis of observational studies. Arch Dis Child Fetal Neonatal Ed. 2018;103(04):F355-F363. Doi: 10.1136/archdischild-2016-312394

25 Kassa AM, Lilja HE. Predictors of postnatal outcome in neonates with gastroschisis. J Pediatr Surg. 2011;46(11):2108-2114. Doi: 10.1016/j.jpedsurg.2011.07.012
26 Lee SL, Beyer TD, Kim SS, et al. Initial nonoperative management and delayed closure for treatment of giant omphaloceles. J Pediatr Surg. 2006;41(11):1846-1849. Doi: 10.1016/j.jpedsurg.2006.06.011

27 Roux N, Jakubowicz D, Salomon L, et al. Early surgical management for giant omphalocele: Results and prognostic factors. J Pediatr Surg. 2018;53(10):1908-1913. Doi: 10.1016/j.jpedsurg.2018.04.036

28 Curry JI, Lander AD, Stringer MDBAPS Multicentre Research Committee. A multicenter, randomized, double-blind, placebo-controlled trial of the prokinetic agent erythromycin in the postoperative recovery of infants with gastroschisis. J Pediatr Surg. 2004;39(04):565-569. Doi: 10.1016/j.jpedsurg.2003.12.020

29 Arnold MA, Chang DC, Nabaweesi R, et al. Risk stratification of 4344 patients with gastroschisis into simple and complex categories. J Pediatr Surg. 2007;42(09):1520-1525. Doi: 10.1016/j. jpedsurg.2007.04.032

30 Heider AL, Strauss RA, Kuller JA. Omphalocele: clinical outcomes in cases with normal karyotypes. Am J Obstet Gynecol. 2004;190 (01):135-141. Doi: 10.1016/j.ajog.2003.06.007

31 Benjamin BG, Ethen MK, Van Hook CL, Myers CA, Canfield MA. Gastroschisis prevalence in Texas 1999-2003. Birth Defects Res A Clin Mol Teratol. 2010;88(03):178-185. Doi: 10.1002/bdra.20642

32 Overcash RT, DeUgarte DA, Stephenson ML, et al; University of California Fetal Consortium* Factors associated with gastroschisis outcomes. Obstet Gynecol. 2014;124(03):551-557. Doi: 10.1097/ aog.0000000000000425

33 van Eijck FC, Wijnen RM, van Goor H. The incidence and morbidity of adhesions after treatment of neonates with gastroschisis and omphalocele: a 30-year review. J Pediatr Surg. 2008;43(03): 479-483. Doi: 10.1016/j.jpedsurg.2007.10.027

34 Lourenço L, Campos M, Monteiro J, et al. Serial transverse enteroplasty (STEP): intermediate outcomes in children with short bowel syndrome. Eur J Pediatr. 2012;171(08):1265-1268. Doi: 10.1007/s00431-012-1728-2

35 Tullie LG, Bough GM, Shalaby A, et al. Umbilical hernia following gastroschisis closure: a common event? Pediatr Surg Int. 2016;32 (08):811-814. Doi: 10.1007/s00383-016-3906-1 